\title{
Direct Gauge Mediation of Uplifted Metastable Supersymmetry Breaking in Supergravity
}

\author{
Nobuhito Maru \\ Department of Physics, Chuo University, Tokyo 112-8551, Japan
}

\begin{abstract}
We propose a direct gauge mediation model based on an uplifted metastable SUSY breaking coupled to supergravity. A constant superpotential plays an essential role to fix the moduli as well as breaking SUSY and R-symmetry and the cancellation of the cosmological constant. Gaugino masses are generated at leading order of SUSY breaking scale, and comparable to the sfermion masses as in the ordinary gauge mediation. Landau pole problem for QCD coupling can be easily solved since more than half of messengers become superheavy, which are heavier than the GUT scale.
\end{abstract}




\section{Introduction}

Supersymmetry (SUSY) is one of the fascinating scenarios solving the hierarchy problem. However, it has to be broken at low energy to be relevant to nature. According to Witten index argument [1], SUSY can be broken nonperturbatively in chiral gauge theories except for the vector-like model in the special case with the color and flavor numbers [2]. Model building by use of chiral gauge theories is not so easy although the nonperturbative dynamics of SUSY theories has been clarified by Seiberg [3]. Several years ago, Intriligator, Seiberg and Shih (ISS) have discovered a metastable SUSY breaking vacuum in (light) massive SUSY QCD in a free magnetic phase [4]. A remarkable fact is that the models they proposed are vector-like models in a wide range of the number of flavors larger than the number of the color. For SUSY breaking model builders, this fact immediately leads to the idea that the Landau pole problem of QCD coupling, which was very hard to solve for a long time, can be easily solved. However, the ISS model has a basic problem that an R-symmetry is not broken in their vacuum, so the (Majorana) gaugino masses cannot be generated after the mediation of SUSY breaking. In order to overcome this problem, there have been many works [5] from various viewpoints by the way to break an R-symmetry explicitly or spontaneously.

Once SUSY and R-symmetry breaking are realized, the next task is to transmit its breaking to our world. Of various mediation mechanisms, the direct gauge mediation [6] is phenomenologically attractive since there is no FCNC problem and it is economical from the model building viewpoint. However, only breaking R-symmetry is not enough and the anomalously small gaugino mass problem has been arisen for the proposed direct gauge mediation models [5] 1. Gaugino masses are not generated at linear order of SUSY breaking scale, but at least generated at the third order. This immediately leads to the fine-tuning in the Higgs mass since the sfermion masses become heavy comparing to the gaugino masses as in the split SUSY scenario [8]. This problem has been recently studied in [9] and the authors have shown that the smallness of gaugino masses is related to the global structure of vacua in renormalizable theories. In particular, it has been shown that models which breaks SUSY in the lowest energy state necessarily lead to anomalously small gaugino masses. To avoid this situation, namely to obtain the gaugino masses at leading order of SUSY breaking, SUSY must be broken in uplifted vacua. Along this direction, several interesting models has been known [10, 11, 12, 13, 14, 15].

In this paper, we propose a direct gauge mediation model based on an uplifted SUSY breaking [11] coupled to supergravity (SUGRA). The model of [11] deforms not only a moduli space such that the rank of the dual quark bilinears is not maximal, but also the mass terms of quarks in an ISS model. Then, SUSY breaking vacuum is uplifted by the deformation and the gaugino masses comparable to the sfermion masses are generated

\footnotetext{
${ }^{1}$ This problem has already been recognized before in $[7$.
} 
as in the ordinary gauge mediation [16]. The model is quite interesting but has some unappealing points as discussed in [11]. The first point is that an explicit R-symmetry breaking term of the moduli has to be introduced since the moduli is undetermined as it stands, and what is worse the overall coefficient is required to be tiny so that the potential have the minimum. The second point is that the perturbative coupling unification is difficult to achieve since the number of messengers is large. This is a similar situation as the direct gauge mediation using the chiral gauge theories These drawbacks are solved in our model by only coupling an uplifted SUSY breaking model [11] to supergravity as follows. R-symmetry breaking is realized by introducing a nonzero constant superpotential with R-charge 2. As a result, the moduli is lifted by the constant superpotential. The magnitude of the constant superpotential is determined by canceling the cosmological constant. Moreover, the Landau pole problem is easily avoided because many of the messengers becomes superheavy, which are larger than the GUT scale.

This paper is organized as follows. In the next section, we discuss our model. We will see that a constant superpotential plays an essential role to lift the moduli as well as canceling the cosmological constant and the breaking of SUSY and R-symmetry. The messenger mass spectrum is calculated in section 3, which is needed in the Landau pole analysis. In section 4, the direct gauge mediation is discussed. We will show that Landau pole problem can be avoided in a wide range of parameters in section 5 . The last section 6 summarizes the results of our paper.

\section{Model}

In this paper, we consider an uplifted metastable SUSY breaking model [1] of IntriligatorSeiberg-Shih (ISS) 4] coupled to supergravity (SUGRA). The uplifted ISS model is a deformed model of an $\mathcal{N}=1$ SUSY $S U(N)\left(N \equiv N_{f}-N_{c}\right)$ gauge theory with $N_{c}+1<$ $N_{f}<\frac{3}{2} N_{c}$ flavor quarks and the gauge singlet couples to the quarks in the superpotential. The matter content and symmetries are summarized as follows.

\begin{tabular}{ccccccc}
\hline & $S U(N)$ & $S U\left(N_{f}\right)$ & $S U\left(N_{f}\right)$ & $U(1)_{B}$ & $U(1)$ & $U(1)_{R}$ \\
\hline$q$ & $\mathbf{N}$ & $\mathbf{N}_{\mathbf{f}}$ & 1 & 1 & 1 & 0 \\
$\tilde{q}$ & $\overline{\mathbf{N}}$ & $\mathbf{1}$ & $\mathbf{N}_{\mathbf{f}}$ & -1 & 1 & 0 \\
$\Phi$ & $\mathbf{1}$ & $\mathbf{N}_{\mathbf{f}}$ & $\overline{\mathbf{N}}_{\mathbf{f}}$ & 0 & -2 & 2 \\
\hline
\end{tabular}

where $S U(N)$ is a gauge group and others are global symmetries. The superpotential and Kähler potential are given by

$$
\begin{aligned}
W & =h q \Phi \tilde{q}-h \mu_{1}^{2} \sum_{i=1}^{k} \Phi_{i}^{i}-h \mu_{2}^{2} \sum_{i=k+1}^{N_{f}} \Phi_{i}^{i}+c, \\
K & =\operatorname{Tr}\left[\Phi^{\dagger} \Phi+q^{\dagger} q+\tilde{q}^{\dagger} \tilde{q}+\cdots\right] .
\end{aligned}
$$


where the trace is taken in flavor space. $c$ is a constant superpotential necessary for the cancellation of the cosmological constant. The ellipsis in the Kähler potential means higher dimensional terms. Note that this model is dual to $\mathcal{N}=1 S U\left(N_{c}\right)$ SUSY gauge theory with $N_{f}$ flavors of quarks $Q$ in the range $N_{c}+1<N_{f}<\frac{3}{2} N_{c}$ and the superpotential

$$
W=m_{1} \sum_{i=1}^{k} Q^{i} \bar{Q}_{i}+m_{2} \sum_{i=k+1}^{N_{f}} Q^{i} \bar{Q}_{i} .
$$

The mass scales are related as $\mu_{1,2}^{2}=-m_{1,2} \Lambda$ where $\Lambda$ is a dynamical scale of the $S U\left(N_{c}\right)$ gauge theory.

Let us first study the vacuum structure of the theory. The classical flat directions are parameterized as follows.

$$
\begin{gathered}
\Phi=\left(\begin{array}{cc}
V_{k \times k} & Y_{k \times\left(N_{f}-k\right)} \\
\tilde{Y}_{\left(N_{f}-k\right) \times k} & Z_{\left(N_{f}-k\right) \times\left(N_{f}-k\right)}
\end{array}\right) \rightarrow\left(\begin{array}{cc}
\mathbf{0}_{k \times k} & \mathbf{0}_{k \times\left(N_{f}-k\right)} \\
\mathbf{0}_{\left(N_{f}-k\right) \times k} & Z_{\left(N_{f}-k\right) \times\left(N_{f}-k\right)}
\end{array}\right), \\
q=\left(\begin{array}{cc}
\left(\chi_{1}\right)_{k \times k} & \left(\rho_{1}\right)_{k \times\left(N_{f}-k\right)} \\
\left(\chi_{2}\right)_{(N-k) \times k} & \left(\rho_{2}\right)_{(N-k) \times\left(N_{f}-k\right)}
\end{array}\right) \rightarrow\left(\begin{array}{cc}
\mu_{1} \mathbf{1}_{k \times k} & \mathbf{0}_{k \times\left(N_{f}-k\right)} \\
\mathbf{0}_{(N-k) \times k} & \mathbf{0}_{(N-k) \times\left(N_{f}-k\right)}
\end{array}\right), \\
\tilde{q}=\left(\begin{array}{cc}
\left(\tilde{\chi}_{1}\right)_{k \times k} & \left(\tilde{\chi}_{2}\right)_{k \times(N-k)} \\
\left(\tilde{\rho}_{2}\right)_{\left(N_{f}-k\right) \times k} & \left(\tilde{\rho}_{2}\right)_{\left(N_{f}-k\right) \times(N-k)}
\end{array}\right) \rightarrow\left(\begin{array}{cc}
\mu_{1} \mathbf{1}_{k \times k} & \mathbf{0}_{k \times(N-k)} \\
\mathbf{0}_{\left(N_{f}-k\right) \times k} & \mathbf{0}_{\left(N_{f}-k\right) \times(N-k)}
\end{array}\right)
\end{gathered}
$$

where $k \leq N$ [11]. For $k=N$, the ISS model is recovered. Using the degrees of freedom of the global symmetries $S U(k) \times S U\left(N_{f}-k\right)$, we find the classical solution as in the right-hand side. The vacuum expectation values $(\mathrm{VEVs})$ of $\chi_{1}, \tilde{\chi}_{1}$ will be determined later by the F-flatness condition.

The VEV of the above fields are determined by the minimization of the scalar potential. In the classical flat directions, the superpotential and the Kähler potential are decomposed as

$$
\begin{aligned}
K= & \operatorname{Tr}\left(|V|^{2}+|\tilde{Y}|^{2}+|Z|^{2}+|Y|^{2}+\left|\chi_{1}\right|^{2}+\left|\chi_{2}\right|^{2}+\left|\tilde{\chi}_{1}\right|^{2}+\left|\tilde{\chi}_{2}\right|^{2}\right) \\
& +\operatorname{Tr}\left(\left|\rho_{1}\right|^{2}+\left|\rho_{2}\right|^{2}+\left|\tilde{\rho}_{1}\right|^{2}+\left|\tilde{\rho}_{2}\right|^{2}+\cdots\right) \\
W= & -h \mu_{2}^{2} \operatorname{Tr} Z+h \sum_{i}^{N-k} Z \rho_{2}^{i}\left(\tilde{\rho}_{2}\right)_{i}+h \sum_{i}^{k}\left[Z \rho_{1}^{i}\left(\tilde{\rho}_{1}\right)_{i}+\mu_{1} \rho_{1}^{i} \tilde{Y}_{i}+\mu_{1} Y^{i}\left(\tilde{\rho}_{1}\right)_{i}\right]+c .
\end{aligned}
$$

It is useful to combine the Kähler potential and the superpotential into a single form

$$
G=K+\ln |W|^{2}
$$

where the Planck scale is set to be the unity. Throughout this paper, this convention is understood.

Using this function $G$, the scalar potential in supergravity is given by

$$
\begin{aligned}
V= & e^{G} \operatorname{Tr}\left[G^{V V^{\dagger}}\left|G_{V}\right|^{2}+G^{Z Z^{\dagger}}\left|G_{Z}\right|^{2}+G^{Y Y^{\dagger}}\left|G_{Y}\right|^{2}+G^{\tilde{Y} \tilde{Y}^{\dagger}}\left|G_{\tilde{Y}}\right|^{2}\right. \\
& +G^{\chi_{1} \chi_{1}^{\dagger}}\left|G_{\chi_{1}}\right|^{2}+G^{\chi_{2} \chi_{2}^{\dagger}}\left|G_{\chi_{2}}\right|^{2}+G^{\tilde{\chi}_{1} \tilde{\chi}_{1}^{\dagger}}\left|G_{\tilde{\chi}_{1}}\right|^{2}+G^{\tilde{\chi}_{2} \tilde{\chi}_{2}^{\dagger}}\left|G_{\tilde{\chi}_{2}}\right|^{2} \\
& \left.+G^{\rho_{1} \rho_{1}^{\dagger}}\left|G_{\rho_{1}}\right|^{2}+G^{\rho_{2} \rho_{2}^{\dagger}}\left|G_{\rho_{2}}\right|^{2}+G^{\tilde{\rho}_{1} \tilde{\rho}_{1}^{\dagger}}\left|G_{\tilde{\rho}_{1}}\right|^{2}+G^{\tilde{\rho}_{2} \tilde{\rho}_{2}^{\dagger}}\left|G_{\tilde{\rho}_{2}}\right|^{2}-3\right] \\
= & e^{K} \operatorname{Tr}\left[\left|h\left(\chi_{1} \tilde{\chi}_{1}-\mu_{1}^{2}\right)\right|^{2}+\left|Z^{\dagger} W-h \mu_{2}^{2}\right|^{2}+\left|\chi_{1}^{\dagger} W\right|^{2}+\left|\tilde{\chi}_{1}^{\dagger} W\right|^{2}-3|W|^{2}\right]
\end{aligned}
$$


where $G_{\phi_{i} \phi_{j}^{\dagger}} \equiv \frac{\partial^{2} G}{\partial \phi_{i} \partial \phi_{j}^{*}}$.

SUSY vacuum conditions are given by

$$
\begin{aligned}
0 & =\chi_{1} \tilde{\chi}_{1}-\mu_{1}^{2} \\
0 & =Z^{\dagger} W-h \mu_{2}^{2}=Z^{\dagger}\left(-h \mu_{2}^{2} \operatorname{Tr} Z+c\right)-h \mu_{2}^{2} \\
0 & =W=-h \mu_{2}^{2} \operatorname{Tr} Z+c
\end{aligned}
$$

which are obviously incompatible, namely SUSY is broken. The vacuum energy is

$$
V_{0}=\operatorname{Tr}\left|F_{Z}\right|^{2}=\left(N_{f}-k\right)\left|h \mu_{2}^{2}\right|^{2}
$$

where $V_{0}$ means the leading terms of the potential independent of the Planck scale.2 At this order $\operatorname{Tr} Z$ is undetermined. On the other hand, the next leading terms of order $\mathcal{O}\left(1 / M_{P}^{2}\right)$ are given by

$$
\begin{aligned}
V_{1} & \approx-h \mu_{2}^{2} \operatorname{Tr}\left(Z^{\dagger} W+Z W^{\dagger}\right)-3|W|^{2}+\left(N_{f}-k\right)\left(2 k \mu_{1}^{2}+\operatorname{Tr}|Z|^{2}\right)\left|h \mu_{2}^{2}\right|^{2} \\
& =-2 h \mu_{2}^{2} \operatorname{Tr} Z\left(-h \mu_{2}^{2} \operatorname{Tr} Z+c\right)-3\left(-h \mu_{2}^{2} \operatorname{Tr} Z+c\right)^{2}+\left(N_{f}-k\right)\left(2 \mu_{1}^{2}+\operatorname{Tr} Z^{2}\right)\left(h \mu_{2}^{2}\right)^{2}
\end{aligned}
$$

where $\langle Z\rangle=\left\langle Z^{\dagger}\right\rangle$ is assumed for simplicity.

Minimization condition

$$
0=\frac{\partial V_{1}}{\partial \operatorname{Tr} Z}=-2 h \mu_{2}^{2}\left[\left(-2 h \mu_{2}^{2} \operatorname{Tr} Z+c\right)-3\left(-h \mu_{2}^{2} \operatorname{Tr} Z+c\right)+\left(N_{f}-k\right) \operatorname{Tr} Z\left(h \mu_{2}^{2}\right)\right]
$$

determines its VEV as

$$
\langle\operatorname{Tr} Z\rangle \approx \frac{2 c}{\left(N_{f}-k+1\right) h \mu_{2}^{2}} .
$$

Note that the moduli is lifted by the constant superpotential $c$ and $U(1)_{R}$ symmetry is spontaneously broken, which is natural because the constant superpotential has an R-charge 2. This should be contrasted with the work [1] in which the $U(1)_{R}$ explicit breaking term $\delta W=Z^{2}$ is added by hand and its overall coefficient must be fine-tuned to be very small to have a minimum. In our case, the fine tuning is reduced to the cancellation of the cosmological constant and it is a generic fine-tuning in the models based on supergravity.

To cancel or obtain a tiny positive cosmological constant, we need the following fine tuning.

$$
\begin{aligned}
0 \approx & V_{0}+V_{1} \approx\left(N_{f}-k\right)\left(h \mu_{2}^{2}\right)^{2}-2 \frac{2 c^{2}}{N_{f}-k+1}\left(-\frac{2}{N_{f}-k+1}+1\right) \\
& -3\left(-\frac{2}{N_{f}-k+1}+1\right)^{2} c^{2}+\left(N_{f}-k\right)\left(h \mu_{2}^{2}\right)^{2}\left[2 k \mu_{2}^{2}+\frac{4 c^{2}}{\left(h \mu_{2}^{2}\right)^{2}\left(N_{f}-k+1\right)^{2}}\right]
\end{aligned}
$$

\footnotetext{
${ }^{2}$ Throughout this paper $e^{K} \simeq e^{|\langle Z\rangle|^{2}} \simeq 1$ is taken by (2.17).
} 
which fixes the cosmological constant

$$
c \approx \sqrt{\frac{N_{f}-k}{3\left(N_{f}-k-1\right)^{2}-4}}\left(N_{f}-k+1\right) h \mu_{2}^{2} .
$$

This leads to

$$
\begin{aligned}
\langle\operatorname{Tr} Z\rangle & \approx 2 \sqrt{\frac{N_{f}-k}{3\left(N_{f}-k-1\right)^{2}-4}}, \\
\langle W\rangle & \approx\left(N_{f}-k-1\right) \sqrt{\frac{N_{f}-k}{3\left(N_{f}-k-1\right)^{2}-4}} h \mu_{2}^{2} .
\end{aligned}
$$

We should understand here the fact that the VEV of the moduli $\operatorname{Tr} Z$ and SUSY breaking scale $F_{\operatorname{Tr}} Z$ are determined by the constant superpotential $c$. Namely, if the vanishing constant superpotential limit $c \rightarrow 0$ is taken, the both of the $\langle\operatorname{Tr} Z\rangle$ and $\left\langle F_{\operatorname{Tr} Z}\right\rangle$ vanish. Therefore, the constant superpotential is essential in our model. From Fig. 1, for our
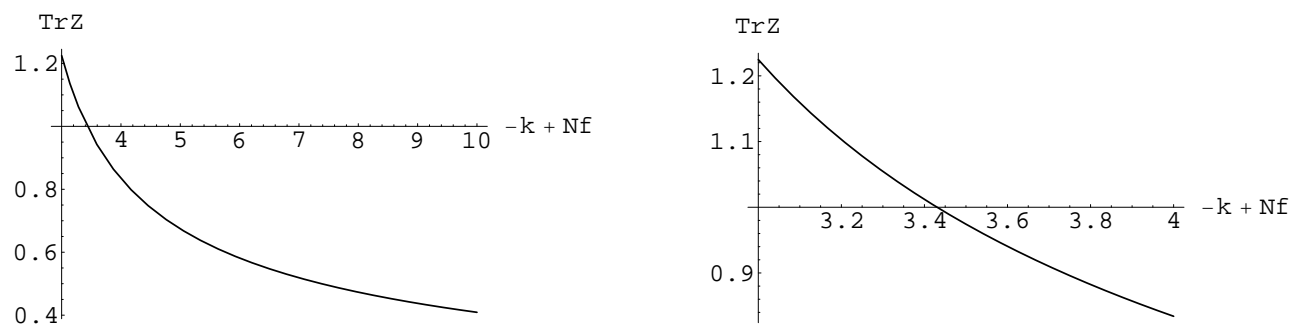

Figure 1: The plot of $\langle\operatorname{Tr} Z\rangle$ as a function of $N_{f}-k$. The plot in the right-hand side is focused on the range around $\langle\operatorname{Tr} Z\rangle=1$ of that in the left-hand side.

analysis to be reliable in the context of effective theories, the moduli VEV must be smaller than the Planck scale $\langle\operatorname{Tr} Z\rangle<1$, which is translated to a condition

$$
N_{f}-k>3.4
$$

This constraint satisfies the assumption $k \leq N=N_{f}-N_{c}$ if $N_{c} \geq 4$.

We comment here on another vacuum with a lower energy than the vacuum discussed in this section. The fields with nonvanishing VEV in another vacuum are

$$
\chi_{1}, \tilde{\chi}_{1} \simeq \mu_{1}, \quad \rho_{1}, \tilde{\rho}_{1} \simeq \mu_{2}
$$

The vacuum energy independent of Planck scale in this vacuum is found to be zero $\left(V_{0}\right)_{\text {another }}=0$. SUSY is broken (SUSY breaking scale is suppressed by Planck scale.). This vacuum is not preferable in the phenomenological viewpoint since the gaugino masses 
via gauge mediation are not generated because of vanishing $\mathrm{VEV}$ of the moduli $\langle Z\rangle=0.3$ Therefore, the decay to this vacuum must be strongly suppressed so that the lifetime of our vacuum be longer than the age of the universe. Let us check that this is indeed the case. We note that the field distance $\Delta \Phi$ and the potential difference $\Delta V$ between our vacuum and another one are $\Delta \Phi \sim\langle\operatorname{Tr} Z\rangle$ and $\Delta V \sim-3 c^{2}$. Therefore, the bounce action between them can be estimated as

$$
S_{\text {bounce }} \sim\left|\frac{(\Delta \Phi)^{4}}{\Delta V}\right| \sim \frac{(\langle\operatorname{Tr} Z\rangle)^{4}}{3 c^{2}} \sim \frac{(\langle\operatorname{Tr} Z\rangle)^{4}}{\left(h \mu_{2}^{2}\right)^{2}} \sim \frac{(\langle\operatorname{Tr} Z\rangle)^{4}}{\left(\left\langle F_{\operatorname{Tr}} Z\right\rangle\right)^{2}} \sim 10^{10-12} \gg \mathcal{O}(100)
$$

where SUSY breaking scale $\left\langle F_{\operatorname{Tr} Z}\right\rangle \approx 10^{10-11} \mathrm{GeV}^{2}$ will be determined later. (2.24) means that the lifetime of our vacuum is longer than the age of universe. Thus, it ensures the stability of our vacuum.

\section{Mass spectrum of messengers}

As will be discussed in the next section, if we embed the Standard Model gauge group into the gauged subgroup of global symmetry $S U\left(N_{f}-k\right)$ in the hidden sector, then the messengers are turned out to be $Z, Y, \tilde{Y}, \rho_{1,2}, \tilde{\rho}_{1,2}$. In this section, we solve the mass spectrum of messengers. The potential for messengers is

$$
\begin{aligned}
V= & e^{K} \operatorname{Tr}\left[\left|V^{\dagger} W+h\left(\chi_{1} \tilde{\chi}_{1}-\mu_{1}^{2}\right)\right|^{2}+\left|Z^{\dagger} W+h\left(\rho_{1} \tilde{\rho}_{1}+\rho_{2} \tilde{\rho}_{2}\right)-h \mu_{2}^{2}\right|^{2}\right. \\
& +\left|Y^{\dagger} W+h\left(\chi_{1} \tilde{\rho}_{1}\right)\right|^{2}+\left|\tilde{Y}^{\dagger} W+h\left(\tilde{\chi}_{1} \rho_{1}\right)\right|^{2} \\
& +\left|\chi_{1}^{\dagger} W+h\left(V \tilde{\chi}_{1}+Y \tilde{\rho}_{1}\right)\right|^{2}+\left|\tilde{\chi}_{1}^{\dagger} W+h\left(V \chi_{1}+Y \rho_{1}\right)\right|^{2} \\
& +\left|\rho_{1}^{\dagger} W+h\left(\tilde{Y} \tilde{\chi}_{1}+Z \tilde{\rho}_{1}\right)\right|^{2}+\left|\tilde{\rho}_{1}^{\dagger} W+h\left(Y \chi_{1}+Z \rho_{1}\right)\right|^{2} \\
& \left.+\left|\rho_{2}^{\dagger} W+h\left(Z \tilde{\rho}_{2}\right)\right|^{2}+\left|\tilde{\rho}_{2}^{\dagger} W+h Z \rho_{2}\right|^{2}+\left|\chi_{2}^{\dagger} W\right|^{2}+\left|\tilde{\chi}_{2}^{\dagger} W\right|^{2}-3|W|^{2}\right] .
\end{aligned}
$$

The nonvanishing VEVs of the curvature of the potential are listed.

$$
\begin{aligned}
\left\langle V_{Z Z^{\dagger}}\right\rangle= & e^{\langle K\rangle}\left[-h \mu_{2}^{2}\left\langle Z^{\dagger} W+Z W^{\dagger}\right\rangle\left(-1+|\langle Z\rangle|^{2}\right)+3\left(h \mu_{2}^{2}\right)^{2}|\langle Z\rangle|^{2}-\left(h \mu_{2}^{2}\right)^{2}\right. \\
& \left.+\left(1+2|\langle Z\rangle|^{2}\right)|\langle W\rangle|^{2}+\left(h \mu_{2}\right)^{2}\left(|\langle\chi\rangle|^{2}+|\langle\tilde{\chi}\rangle|^{2}\right)\left\langle Z+Z^{\dagger}\right\rangle\right] \\
\approx & -\left(h \mu_{2}^{2}\right)^{2}-4 h \mu_{2}^{2} c\langle Z\rangle+|\langle W\rangle|^{2} \approx\left(h \mu_{2}^{2}\right)^{2} \\
\left\langle V_{Y Y^{\dagger}}\right\rangle= & \left\langle V_{\tilde{Y} \tilde{Y}^{\dagger}}\right\rangle=e^{\langle K\rangle}\left[\left(h \mu_{1}\right)^{2}+|\langle W\rangle|^{2}\right] \approx\left(h \mu_{1}\right)^{2} \\
\left\langle V_{\rho_{1} Y^{\dagger}}\right\rangle= & \left\langle V_{Y \rho_{1}^{\dagger}}\right\rangle=e^{\langle K\rangle} h^{2} \mu_{1}\langle Z\rangle \\
\left\langle V_{\tilde{\rho}_{1} \tilde{Y}^{\dagger}}\right\rangle= & \left\langle V_{\tilde{Y} \tilde{\rho}_{1}^{\dagger}}\right\rangle=e^{\langle K\rangle} h^{2} \mu_{1}\langle Z\rangle^{\dagger} \\
\left\langle V_{\rho_{1} \rho_{1}^{\dagger}}\right\rangle= & \left\langle V_{\tilde{\rho}_{1} \tilde{\rho}_{1}^{\dagger}}\right\rangle=e^{\langle K\rangle}\left[(h\langle Z\rangle)^{2}+|\langle W\rangle|^{2}+\left(h \mu_{1}\right)^{2}\right] \approx e^{\langle K\rangle}(h\langle Z\rangle)^{2}, \\
\left\langle V_{\rho_{2} \rho_{2}^{\dagger}}\right\rangle= & \left\langle V_{\tilde{\rho}_{2} \tilde{\rho}_{2}^{\dagger}}\right\rangle=e^{\langle K\rangle}\left[(h\langle Z\rangle)^{2}+|\langle W\rangle|^{2}\right] \approx e^{\langle K\rangle}(h\langle Z\rangle)^{2} .
\end{aligned}
$$

\footnotetext{
${ }^{3}$ Note that R-symmetry is broken by the constant superpotential. In principle, the gravity mediated gaugino masses are of order $\langle W\rangle / M_{P}^{2} \sim \mathrm{TeV}$ at tree level. However, as will be described later in the text, we assume throughout this paper that the hidden and the visible sectors are sequestered in the superspace density to avoid flavor dependent sfermion masses at tree level. As a result, the gravity mediated gaugino masses are not generated at tree level as well. Although the sizable gaugino masses can be obtained via anomaly mediation, we does not consider this possibility in this paper.
} 
In this estimation, the terms suppressed by the Planck scale are simply neglected or $h\langle Z\rangle \gg h \mu_{1}$ is considered. It is easy to find the masses of $Z, \rho_{2}, \tilde{\rho}_{2}$ since they have no mixing. If we take into account SUSY breaking mass for $\rho_{1,2}, \tilde{\rho}_{1,2}$, the mixing takes place from the terms

$$
V \supset h F_{\operatorname{Tr} Z}\left(\rho_{1} \tilde{\rho}_{1}+\rho_{2} \tilde{\rho}_{2}\right)+\text { h.c. }
$$

then the mass terms of $\rho_{2}, \tilde{\rho}_{2}$ sector are read off

$$
\left(\rho_{2}^{\dagger}, \tilde{\rho}_{2}\right)\left(\begin{array}{cc}
|M|^{2} & \left(h F_{\operatorname{Tr} Z}\right)^{\dagger} \\
\left(h F_{\operatorname{Tr} Z}\right) & |M|^{2}
\end{array}\right)\left(\begin{array}{c}
\rho_{2} \\
\tilde{\rho}_{2}^{\dagger}
\end{array}\right)
$$

where $|M|^{2} \equiv e^{\langle K\rangle}|\langle W\rangle|^{2}+|h\langle\operatorname{Tr} Z\rangle|^{2}$. The eigenvalues are

$$
m_{\rho_{2}^{ \pm}}^{2}=e^{\langle K\rangle}\left[|M|^{2} \pm h\left\langle F_{\operatorname{Tr} Z}\right\rangle\right] \approx(h\langle\operatorname{Tr} Z\rangle)^{2} \approx \mathcal{O}\left(\left(0.1 M_{P}\right)^{2}\right)
$$

and the corresponding eigenstates are $\rho_{2}^{ \pm} \equiv\left(\rho_{2} \pm \tilde{\rho}_{2}^{\dagger}\right) / \sqrt{2}$. The approximation $(h\langle Z\rangle)^{2} \gg$ $\left\langle F_{\operatorname{Tr} Z}\right\rangle$ is taken, which will be justified later.

The mass matrix for $Y, \tilde{Y}, \rho_{1}, \tilde{\rho}_{1}$ before SUSY breaking is found to be

$$
\left(Y^{\dagger}, \rho_{1}^{\dagger}, \tilde{Y}^{\dagger}, \tilde{\rho}_{1}^{\dagger}\right)\left(\begin{array}{cccc}
m_{|Y|^{2}}^{2} & m_{Y^{\dagger} \rho_{1}}^{2} & 0 & 0 \\
m_{\rho_{1}^{\dagger} Y}^{2} & m_{\left|\rho_{1}\right|^{2}}^{2} & 0 & 0 \\
0 & 0 & m_{|\tilde{Y}|^{2}}^{2} & m_{\tilde{Y}^{\dagger} \tilde{\rho}_{1}}^{2} \\
0 & 0 & m_{\tilde{Y} \tilde{\rho}_{1}^{\dagger}}^{2} & m_{\left|\tilde{\rho}_{1}\right|^{2}}^{2}
\end{array}\right)\left(\begin{array}{c}
Y \\
\rho_{1} \\
\tilde{Y} \\
\tilde{\rho}_{1}
\end{array}\right)
$$

where

$m_{|Y|^{2}}^{2}=m_{|\tilde{Y}|^{2}}^{2} \approx\left(h \mu_{1}\right)^{2}, \quad m_{Y^{\dagger} \rho_{1}}^{2}=m_{\tilde{Y}^{\dagger} \tilde{\rho}_{1}}^{2} \approx h^{2} \mu_{1}\langle\operatorname{Tr} Z\rangle, \quad m_{\left|\rho_{1}\right|^{2}}^{2}=m_{\left|\tilde{\rho}_{1}\right|^{2}}^{2} \approx(h\langle\operatorname{Tr} Z\rangle)^{2}$.

Diagonalizing the mass matrix in $Y$ - $\rho_{1}$ sector, we obtain eigenvalues $(h\langle\operatorname{Tr} Z\rangle)^{2}$ or $\left(h \mu_{1}\right)^{2}$ and the corresponding eigenmodes are

$$
\hat{\tilde{\rho}}_{1} \equiv \tilde{\rho}_{1}+\frac{\mu_{1}}{\langle\operatorname{Tr} Z\rangle} \tilde{Y}, \quad \hat{\tilde{Y}} \equiv \tilde{Y}-\frac{\mu_{1}}{\langle\operatorname{Tr} Z\rangle} \tilde{\rho}_{1} .
$$

In the above calculation, we assumed no accidental cancellation among $\mathcal{O}(1)$ coefficients. A similar calculation for $\tilde{Y}$ - $\tilde{\rho}_{1}$ sector is also applied. Taking into account SUSY breaking for $\rho_{1}, \tilde{\rho}_{1}$ sector, the leading order mass matrix for $\hat{\rho}_{1}, \hat{\tilde{\rho}}_{1}$ sector is identical to the one for $\rho_{2}, \tilde{\rho}_{2}$ sector. Therefore, the mass eigenvalues and its corresponding mass eigenstates are

$$
\begin{aligned}
& m_{\hat{\rho}_{1}^{ \pm}}=e^{\langle K\rangle}\left[(h\langle\operatorname{Tr} Z\rangle)^{2} \pm h\left\langle F_{\operatorname{Tr} Z}\right\rangle\right] \approx(h\langle\operatorname{Tr} Z\rangle)^{2}, \\
& \hat{\rho}_{1}^{ \pm} \equiv\left(\hat{\rho}_{1} \pm \hat{\tilde{\rho}}_{1}^{\dagger}\right) / \sqrt{2} .
\end{aligned}
$$

On the other hand, the masses of messenger fermions are read from the superpotential [11]

$$
W=-h \mu_{2}^{2} \operatorname{Tr} Z+h \sum_{i=1}^{N-k} \operatorname{Tr} Z \rho_{2}^{i}\left(\tilde{\rho}_{2}\right)_{i}+h \sum_{i=1}^{k}\left[\operatorname{Tr} Z \rho_{1}^{i}\left(\tilde{\rho}_{1}\right)_{i}+\mu_{1} \rho_{1}^{i} \tilde{Y}_{i}+\mu_{1} Y^{i}\left(\tilde{\rho}_{1}\right)_{i}\right]
$$


as

$$
h\left(\psi_{\tilde{\rho}_{1}}, \psi_{\tilde{Y}}, \psi_{\tilde{\rho}_{2}}\right) \underbrace{\left(\begin{array}{ccc}
\operatorname{Tr} Z & \mu_{1} & 0 \\
\mu_{1} & 0 & 0 \\
0 & 0 & \operatorname{Tr} Z
\end{array}\right)}_{\equiv \mathcal{M}}\left(\begin{array}{c}
\psi_{\rho_{1}} \\
\psi_{Y} \\
\psi_{\rho_{2}}
\end{array}\right) .
$$

$\psi_{\rho_{2}}$ is decouple and the mass eigenvalues of $\rho_{1}-Y$ sector is obtained as $h\langle\operatorname{Tr} Z\rangle$ and $h \mu_{1}$. We see that some (the other) linear combination of messenger multiplets in $\rho_{1}-Y$ sector is heavy (light) with mass $h\langle\operatorname{Tr} Z\rangle\left(h \mu_{1}\right)$.

Note that the fermionic component of $Z$ in the adjoint representation is massless at tree level. However, it will be massive at one-loop level through the ordinary gauge-mediated diagram.

$$
m_{\psi_{Z}} \simeq \frac{\alpha_{h}}{4 \pi} \frac{\left\langle F_{\operatorname{Tr} Z}\right\rangle}{\langle\operatorname{Tr} Z\rangle} \sim 0.01 \times \frac{\left(10^{11} \mathrm{GeV}\right)^{2}}{0.1 M_{P} \mathrm{GeV}} \sim \mathcal{O}(\mathrm{TeV}) .
$$

Trace part of fermionic component of $Z$ corresponds to NG fermion, which is absorbed into the longitudinal component of the gravitino after SUSY breaking.

\section{Direct Gauge Mediation}

Gauging a subgroup $S U\left(N_{f}-k\right)$ of the unbroken flavor symmetry $S U(k) \times S U\left(N_{f}-\right.$ $k$ ) of the model, SUSY breaking is transmitted to the MSSM through the messenger $Y, \tilde{Y}, \rho_{1,2}, \tilde{\rho}_{1,2}$ loops. In the uplifted ISS model, the superpotential for messengers takes of the form (3.16). The second term in the $\rho_{2}, \tilde{\rho}_{2}$ sector corresponds to the messenger interactions in the minimal gauge mediation, which gives contributions to both gaugino and sfermion masses. However, the last three terms in the $Y-\rho_{1}$ sector provide contributions to the sfermion masses only as in the ISS model.

Gaugino and sfermion masses are calculated in [17].

$$
\begin{aligned}
M_{r} & =\frac{\alpha_{r}}{4 \pi} \Lambda_{G}, \\
m^{2} & =2 \sum_{r=1}^{3} C_{r}\left(\frac{\alpha_{r}}{4 \pi}\right)^{2} \Lambda_{S}^{2}
\end{aligned}
$$

where $r=1,2,3$ standing for $U(1), S U(2), S U(3)$, respectively. $\quad C_{r}$ is the quadratic Casimir in the gauge group corresponding to $r$.

$$
\begin{aligned}
\Lambda_{G} & \equiv\left\langle F_{\operatorname{Tr} Z}\right\rangle \partial_{\operatorname{Tr} Z} \log \operatorname{det} \mathcal{M}, \\
\Lambda_{S}^{2} & \equiv \frac{1}{2}\left|\left\langle F_{\operatorname{Tr} Z}\right\rangle\right|^{2} \frac{\partial^{2}}{\partial \operatorname{Tr} Z^{\dagger} \partial \operatorname{Tr} Z} \sum_{i=1}^{N}\left(\log |\mathcal{M}|^{2}\right)^{2} .
\end{aligned}
$$


$Z$ dependent mass matrix $\mathcal{M}$ is given in (3.17). Using this $\mathcal{M}$, we obtain the gaugino and sfermion masses

$$
\begin{aligned}
M_{r}= & \frac{\alpha_{r}}{4 \pi}(N-k) \frac{\left\langle F_{\mathrm{Z}}\right\rangle}{\langle\operatorname{Tr} Z\rangle} \approx \frac{\alpha_{r}}{4 \pi}(N-k) \frac{h \mu_{2}^{2}}{0.1 M_{P}} \\
m^{2}= & 2 \sum_{r=1}^{3} C_{r}\left(\frac{\alpha_{r}}{4 \pi}\right)^{2}\left|\left\langle F_{\operatorname{Tr} Z}\right\rangle\right|^{2} \times \\
& {\left[\frac{N-k}{|\langle\operatorname{Tr} Z\rangle|^{2}}+\frac{2 k}{|\langle\operatorname{Tr} Z\rangle|^{2}+4 \mu_{1}^{2}}+\frac{2 k \log \left(\frac{|\langle\operatorname{Tr} Z\rangle|^{2}+2 \mu_{1}^{2}+|\langle\operatorname{Tr} Z\rangle| \sqrt{|\langle\operatorname{Tr} Z\rangle|^{2}+4 \mu_{1}^{2}}}{|\langle\operatorname{Tr} Z\rangle|^{2}+2 \mu_{1}^{2}-|\langle\operatorname{Tr} Z\rangle| \sqrt{|\langle\operatorname{Tr} Z\rangle|^{2}+4 \mu_{1}^{2}}}\right)}{\left(|\langle\operatorname{Tr} Z\rangle|^{2}+4 \mu_{1}^{2}\right) \sqrt{|\langle\operatorname{Tr} Z\rangle|^{4}+4 \mu_{1}^{2}|\langle\operatorname{Tr} Z\rangle|^{2}}}\right] } \\
\approx & 2 \sum_{r=1}^{3} C_{r}\left(\frac{\alpha_{r}}{4 \pi}\right)^{2}(N+k)\left(\frac{h \mu_{2}^{2}}{\langle\operatorname{Tr} Z\rangle}\right)^{2} \approx\left(\frac{\alpha_{r}}{4 \pi}\right)^{2}\left(\frac{h \mu_{2}^{2}}{0.1 M_{P}}\right)^{2}
\end{aligned}
$$

where $\langle\operatorname{Tr} Z\rangle \gg \mu_{1}$ are taking into account in the sfermion masses. Here we note that the gaugino and the sfermion masses are comparable (up to $\mathcal{O}(1)$ constant) similar to the ordinary gauge mediation case.

Requiring $M_{r} \simeq m$ to be $1 \mathrm{TeV}$, we obtain

$$
(N-k) h \mu_{2}^{2} \approx 10^{4} M_{P} \rightarrow\left\langle F_{\text {TrZ }}\right\rangle=h \mu_{2}^{2} \approx\left(10^{10 \sim 11} \mathrm{GeV}\right)^{2}
$$

This leads to the gravitino mass as

$$
m_{3 / 2}=\frac{\left\langle F_{\operatorname{Tr} Z}\right\rangle}{\sqrt{3} M_{P}} \approx \frac{10^{4} M_{P}}{\sqrt{3} M_{P}} \simeq 1 \mathrm{TeV} .
$$

This result means that the gravity mediation effects to the sfermion masses are comparable to the gauge mediation one. This is an undesirable feature since the gravity mediation to the sfermion masses are generated at tree level from the flavor dependent contact term $\int d^{4} \theta Z^{\dagger} Z Q_{i}^{\dagger} Q_{j}(i, j$ : flavor indices, $Q$ : the MSSM multiplets) and cause the large flavor violation at unacceptable level in general. Therefore, we need to "sequester" the hidden sector from the visible sector at tree level. This can be realized if the hidden sector and the visible sector are separated in the superspace density $\varphi$ not in the Kähler potential [18. Namely,

$$
\varphi=\varphi_{h}\left(Z^{\dagger}, Z\right)+\varphi_{v}\left(Q^{\dagger}, Q\right)
$$

The superspace density is related to the Kähler potential as $K=-3 \ln \varphi$. As a result, the gravity mediated sfermion masses vanish at tree level and the next leading masses are generated by anomaly mediation if the compensator multiplet has an F-term VEV. Throughout this paper, we assume the form of the superspace density (4.9) and the compensator does not develop F-term VEV for simplicity because our focus is on the direct gauge mediation. 


\section{Landau pole analysis}

In this model, there are extra multiplets with the SM charges (i.e. messengers) other than the MSSM multiplets, namely adjoint representation of $Z$, and the (anti) fundamental ones $Y, \tilde{Y}, \rho_{1,2}, \tilde{\rho}_{1,2}$. As was calculated before, the masses of $\rho_{2}, \tilde{\rho}_{2}$ and $\rho_{1}+\frac{h \mu_{1}}{h\langle Z\rangle} Y, \tilde{\rho}_{1}+\frac{h \mu_{1}}{h\langle Z\rangle} \tilde{Y}$ are $\mathcal{O}\left(0.1 M_{P}\right)>M_{\mathrm{GUT}}$, so these multiplets do not affect the gauge coupling unification. On the other hand, the multiplets $Y-\frac{h \mu_{1}}{h\langle Z\rangle} \rho_{1}, \tilde{Y}-\frac{h \mu_{1}}{h\langle Z\rangle} \tilde{\rho}_{1}$ have masses of order $h \mu_{1} . Z$ have a mass of order $\mathcal{O}(\mathrm{TeV})$, which have to be taken into account in the gauge coupling running. As will be shown below, the Landau pole problem for QCD coupling can be easily avoided comparing to the model of [11] since more than half of messengers become superheavy of order $0.1 M_{P}$.

The mass spectrum is summarized below.

$$
\begin{aligned}
& m_{\hat{\rho}_{1}}, m_{\hat{\tilde{\rho}}_{1}}, m_{\rho_{2}}, m_{\tilde{\rho}_{2}} \approx 0.1 M_{P}, \\
& m_{\hat{Y}}, m_{\hat{\tilde{Y}}} \approx h \mu_{1}, \quad m_{\lambda} \simeq m_{\tilde{f}} \approx m_{Z} \approx 1 \mathrm{TeV} .
\end{aligned}
$$

Now, we study a Landau pole constraint for QCD gauge coupling. We consider the two cases where the messengers are embedded into the MSSM multiplets and the SU(5) GUT multiplets.

One-loop gauge coupling RGE is given by

$$
g_{i}^{-2}(\mu)=g_{i}^{-2}\left(\mu^{\prime}\right)+\frac{b_{i}}{8 \pi^{2}} \ln \left(\frac{\mu}{\mu^{\prime}}\right)
$$

where $\mu, \mu^{\prime}$ are the renormalization scales. $b_{i}$ is one-loop beta function coefficient of the gauge group $i=S U(3), S U(2), U(1)$.

First, we study the MSSM embedding case. The one-loop beta function coefficients for QCD coupling at various scales are listed below.

$$
\begin{array}{cl}
m_{W}<\mu<\mathrm{TeV} \quad: \quad b_{3}=b_{3}^{\mathrm{SM}}=7 \\
\mathrm{TeV}<\mu<h \mu_{1} \quad: \quad b_{3}=b_{3}^{\mathrm{MSSM}}-\underbrace{b_{3}(Z)}_{3}=0 \\
h \mu_{1}<\mu<h\langle Z\rangle \approx \mathcal{O}\left(0.1 M_{P}\right) \quad: \quad b_{3}=b_{3}^{\mathrm{MSSM}}-b_{3}(Z)-\underbrace{b_{3}(\hat{Y}, \hat{\tilde{Y}})}_{k \times(1 / 2+1 / 2)}=-k
\end{array}
$$

where $b_{3}^{\mathrm{SM}}, b_{3}^{\mathrm{MSSM}}$ are the QCD one-loop beta function coefficients for the standard model and the minimal SUSY standard model.

The requirement that the QCD coupling at the GUT scale is perturbative

$$
\alpha_{3}\left(M_{\mathrm{GUT}}\right)=\frac{1}{\alpha_{3}\left(m_{W}\right)^{-1}-\frac{1}{2 \pi}\left[7 \ln \left(m_{W} / \mathrm{TeV}\right)-k \ln \left(h \mu_{1} / M_{\mathrm{GUT}}\right)\right]}<1
$$

constrains the number of messengers $k$ and the mass scale $h \mu_{1}$ as

$$
x>16-\frac{27.5}{k} .
$$


where we made use of $\alpha_{3}\left(m_{W}\right) \simeq 0.118$ and assumed $h \mu_{1} \equiv 10^{x} \mathrm{GeV}$ to be smaller than the GUT scale.

Similarly, noting that extra multiplets $(\mathbf{3}, \mathbf{2}),\left(\mathbf{3}^{*}, \mathbf{2}\right)$ with the SM charges from $Z$ multiplet contribute to the QCD gauge coupling running in the GUT embedding case, we obtain a similar constraint

$$
x>16-\frac{14.5}{k} .
$$

As an illustration, we show plots of (5.5) and (5.6) in Fig. 2. Here we assume the mass
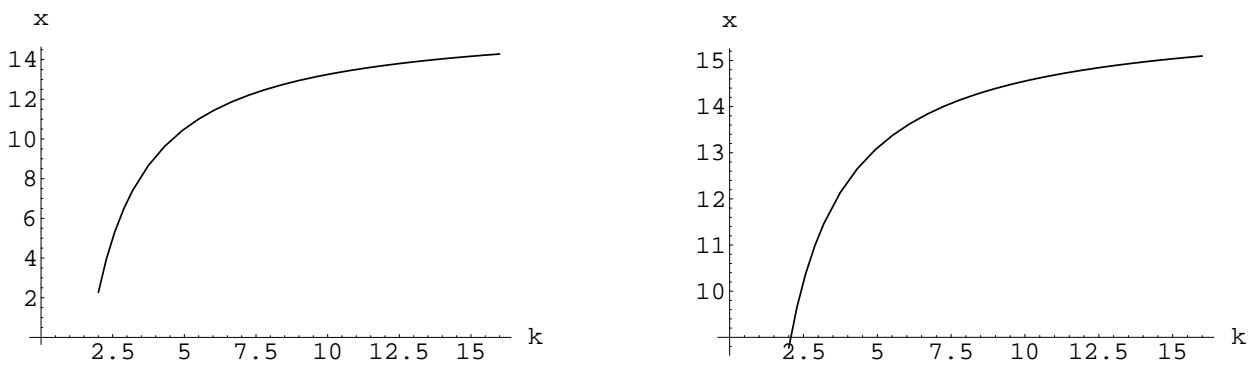

Figure 2: The plot of constraints for the mass scale $h \mu_{1}$ as a function of the number of messengers $k$, the MSSM case (5.5) (left) and the GUT case (5.6) (right).

scale $h \mu_{1}$ to be larger than TeV since the messengers $\hat{Y}, \hat{\tilde{Y}}$ have not been observed below the $\mathrm{TeV}$ scale. The upper region of the plot implies that the perturbative unification is possible. More concretely, some numerical examples allowed by corresponding constraints are listed in the following Table.

\begin{tabular}{|c|c|c|}
\hline$k$ & $x($ MSSM $)$ & $x($ GUT $)$ \\
\hline 1 & No constraint & No constraint \\
\hline 2 & No constraint & $>8.77$ \\
\hline 3 & $>6.84$ & $>11.1$ \\
\hline 4 & $>9.13$ & $>12.4$ \\
\hline
\end{tabular}

If $h \mu_{1}>M_{\mathrm{GUT}}$, only the $Z$ multiplet in the adjoint representation contributes to the QCD gauge coupling running. In this case, we can easily check that the perturbative unification is possible without any problem.

\section{Summary}

In this paper, we have discussed a model of direct gauge mediation based on an uplifted metastable SUSY breaking model in supergravity. The constant superpotential plays an essential role to fix the moduli as well as breaking SUSY and R-symmetry and the cancellation of the cosmological constant. SUSY breaking is directly gauge mediated to superparticles by gauging a subgroup of unbroken flavor symmetry. In particular, the 
gaugino masses are generated at leading order of SUSY breaking scale, namely comparable

to the sfermion masses as in the ordinary gauge mediation. Improvements by simply coupling an uplifted metastable SUSY breaking model [1] to supergravity are two fold. One is that the ad hoc fine-tuning to fix the moduli in the model of [11] reduces to the fine-tuning to cancel the cosmological constant necessary and generic for any model based on supergravity The other is that Landau pole problem for QCD coupling can be easily solved since more than half of messengers become superheavy, which are heavier than the GUT scale. The constraint for the number of messengers and their mass scale is obtained by the effects of the remaining light messengers to the running of QCD coupling constant.

In our analysis, the anomaly mediation contribution is simply neglected by assuming a vanishing F-term of the compensator since our main interests are focused on the direct gauge mediation. Relaxing this assumption, the pattern of superparticle spectrum would become quite rich by the interplay of gauge and anomaly mediations. Studying such a spectrum in detail will be left for a future work.

\section{Acknowledgments}

The work of the author was supported in part by the Grant-in-Aid for Scientific Research of the Ministry of Education, Science and Culture, No.18204024.

\section{References}

[1] E. Witten, Nucl. Phys. B 188, 513 (1981); Nucl. Phys. B 202, 253 (1982).

[2] K. I. Izawa and T. Yanagida, Prog. Theor. Phys. 95, 829 (1996); K. A. Intriligator and S. D. Thomas, Nucl. Phys. B 473, 121 (1996).

[3] N. Seiberg, Nucl. Phys. B 435, 129 (1995), Phys. Rev. D 49, 6857 (1994).

[4] K. Intriligator, N. Seiberg and D. Shih, JHEP 0604, 021 (2006).

[5] C. Csaki, Y. Shirman and J. Terning, JHEP 0705, 099 (2007); S. Abel, C. Durnford, J. Jaeckel and V. V. Khoze, Phys. Lett. B 661, 201 (2008); N. Haba and N. Maru, Phys. Rev. D 76, 115019 (2007); N. Haba, JHEP 0803, 059 (2008); Z. Lalak and O. J. Eyton-Williams, JHEP 0903, 147 (2009); R. Essig, J. F. Fortin, K. Sinha, G. Torroba and M. J. Strassler, JHEP 0903, 043 (2009).

[6] E. Poppitz and S. P. Trivedi, Phys. Rev. D 55, 5508 (1997); N. Arkani-Hamed, J. March-Russell and H. Murayama, Nucl. Phys. B 509, 3 (1998); H. Murayama, Phys. Rev. Lett. 79, 18 (1997); S. Dimopoulos, G. R. Dvali, R. Rattazzi and G. F. Giudice, Nucl. Phys. B 510, 12 (1998); N. Okada, Phys. Lett. B 423, 305 
(1998); K. Agashe, Phys. Lett. B 435, 83 (1998); N. Maru, Phys. Lett. B 436, 311 (1998).

[7] K. I. Izawa, Y. Nomura, K. Tobe and T. Yanagida, Phys. Rev. D 56, 2886 (1997).

[8] N. Arkani-Hamed and S. Dimopoulos, JHEP 0506, 073 (2005).

[9] Z. Komargodski and D. Shih, JHEP 0904, 093 (2009).

[10] R. Kitano, H. Ooguri and Y. Ookouchi, Phys. Rev. D 75, 045022 (2007).

[11] A. Giveon, A. Katz and Z. Komargodski, JHEP 0907, 099 (2009).

[12] S. A. Abel, J. Jaeckel and V. V. Khoze, Phys. Lett. B 682, 441 (2010).

[13] J. Barnard, JHEP 1002, 035 (2010).

[14] R. Auzzi, S. Elitzur and A. Giveon, JHEP 1003, 094 (2010).

[15] K. Hanaki and Y. Ookouchi, arXiv:1003.5663 [hep-ph].

[16] M. Dine, W. Fischler and M. Srednicki, Nucl. Phys. B 189, 575 (1981); S. Dimopoulos and S. Raby, Nucl. Phys. B 192, 353 (1981); M. Dine and W. Fischler, Phys. Lett. B 110, 227 (1982); Nucl. Phys. B 204, 346 (1982); C. R. Nappi and B. A. Ovrut, Phys. Lett. B 113, 175 (1982); L. Alvarez-Gaume, M. Claudson and M. B. Wise, Nucl. Phys. B 207, 96 (1982); S. Dimopoulos and S. Raby, Nucl. Phys. B 219, 479 (1983); M. Dine and A. E. Nelson, Phys. Rev. D 48, 1277 (1993); M. Dine, A. E. Nelson and Y. Shirman, Phys. Rev. D 51, 1362 (1995); M. Dine, A. E. Nelson, Y. Nir and Y. Shirman, Phys. Rev. D 53, 2658 (1996).

[17] C. Cheung, A. L. Fitzpatrick and D. Shih, JHEP 0807, 054 (2008).

[18] K. Inoue, M. Kawasaki, M. Yamaguchi and T. Yanagida, Phys. Rev. D 45, 328 (1992) 